\title{
Quantum Mechanical Tunneling of Dislocations: Quantization and Depinning from Peierls Barrier
}

\author{
Saleem Iqbal1, Farhana Sarwar $^{2}$, Syed Mohsin Raza ${ }^{3}$ \\ ${ }^{1}$ Department of Mathematics, University of Balochistan, Quetta, Pakistan \\ ${ }^{2}$ Department of Mathematics, F. G. Girls Degree College, Quetta, Pakistan \\ ${ }^{3}$ Department of Physics, University of Balochistan, Quetta, Pakistan \\ Email:smraza7@yahoo.com
}

Received 2 February 2016; accepted 14 May 2016; published 17 May 2016

Copyright (C) 2016 by authors and Scientific Research Publishing Inc.

This work is licensed under the Creative Commons Attribution International License (CC BY). http://creativecommons.org/licenses/by/4.0/

(c) (i) Open Access

\begin{abstract}
Theories of Mott and Weertmann pertaining to quantum mechanical tunneling of dislocations from Peierls barrier in cubic crystals are revisited. Their mathematical calculations about logarithmic creep rate and lattice vibrations as a manifestation of Debye temperature for quantized thermal energy are found correct but they can not ascertain to choose the mass of phonon or "quanta" of lattice vibrations. The quantum mechanical yielding in metals at relatively low temperatures, where Debye temperatures operate, is resolved and the mathematical formulas are presented. The crystal plasticity is studied with stress relaxation curves instead of logarithmic creep rate. With creep rate formulas of Mott and Weertmann, a new formula based on logarithmic profile of stress relaxation curves is proposed which suggests simultaneous quantization of dislocations with their stress, i.e., $\ln \hbar \sigma$ and depinning of dislocations, i.e., $\ln (N A / t)$, where $\hbar$ is quantum action, $\sigma$ is the stress, $N$ is the number of dislocations, $A$ is the area and $t$ is the time. The two different interpretations of "quantum length of Peierls barrier", one based on curvature of space, i.e., $\partial / \partial r$ yields quantization of Burgers vector and the other based on the curvature of time, i.e., $\partial / \partial t$ yields depinning of dislocations from Peierls barrier in cubic crystals, are presented. $(\partial / \partial t) \eta$, i.e., the unitary operator on shear modulus yields the variations in the curvature of time due to which simultaneous quantization, and depinning of dislocations occur from Peierls barrier in cubic crystals.
\end{abstract}

\section{Keywords}

Peierls Barrier, Quantum Tunneling, Dislocations, Stress Relaxation, Quantum of Stresses, Depinning of Dislocations 


\section{Introduction}

With the advent of quantization of the motion of dislocations due to lattice vibrations by Mott [1], a new idea following the same treatment to the case of dislocations crossing Peierls barrier was floated by Weertmann [2]. Dislocations may advance after depinning from the Peierls barrier by quantum mechanical tunneling, advancing on front of $2 n$ atoms. The rigorous mathematical calculations by them [1] [2] are avoided. Only the results are expressed for providing meaningful interpretations and indeed modifications. The rate of successful barrier crossings is

$$
\left(\frac{c}{4 n a}\right) \exp \left\{-10.4\left(M u_{o}\right)^{1 / 2} \hbar^{-1} a \eta\right\}
$$

where the frequency is $v=\frac{c}{4 n a} \equiv \frac{c}{\lambda}, a$ is the lattice parameter, $M$ is the mass of the ion core, $\hbar$ is the Planck's constant, $u_{o}$ is the activation energy and $\eta$ is the shear modulus. The logarithmic creep, as suggested by Weertmann [2] following Mott [1] is

$$
\dot{\varepsilon}=\frac{N \hbar \sigma A}{10.4\left(M u_{o}\right)^{1 / 2} t} .
$$

$\sigma$ is the stress, where $N$ is the number of dislocations, $A$ is the activation area, $\dot{\varepsilon}$ is the creep rate and $\hbar$ is Planck's constant. They suggested that the quantum mechanical activated creep will be more rapid than the thermally activated creep. They approximated $\left\{\frac{\hbar}{b\left(M u_{o}\right)^{1 / 2}}\right\}^{3 / 2} \approx\left\{\frac{m}{M}\right\}^{1 / 2}$ and considered $u_{o} \approx \frac{\hbar^{2}}{m b^{2}}$ where $m$ is the mass of an electron and $b$ is the Burgers vector. Their original formulation for quantizing the motion of dislocations with lattice vibrations contradicts the approximation for mass of the electron. They would have considered the quanta of lattice vibrations by considering the mass of the phonon. The mass of the phonon can be determined by knowing the frequency of phonon under damped conditions (Bardoni resonant peaks) for depinning of dislocations from Peierls barrier. Using $E=h v=m c^{2}$, the mass of phonon from Bardoni peak, can be obtained. But, their approximation for classical thermal energy, $k T$ (where $k$ is Boltzmann constant and $T$ is the temperature in kelvin) by considering temperature in the form of Debye temperature (follows Debye theory of specific heat of crystals and is based on $T^{3}$ variation, i.e., a quantum theory of lattice vibrations) is the most validated result for quantum behavior of thermal energy, i.e.,

$$
k T \equiv k \Theta \approx \hbar\left(\frac{u_{o}}{m b^{2}}\right)^{1 / 2}
$$

where $m$ will be considered for the mass of phonon instead of electron and $b$ is the Bourger's vector. where $\Theta$ is Debye temperature

The quantum mechanical effects in a single barrier stochastic model were allowed for stress relaxation [3] [4]. The model for stress relaxation, at low temperatures, was proposed [5]. This suggested that the behaviour of stress relaxation was "athermal” (quantum mechanical) and logarithmic in character. A self consistent stress relaxation model was developed [6] which indicated that the strain rate sensitivity of the relaxation rate for each stress relaxation curve during work hardening was congruent to slope of the logarithm creep rate, i.e.,

$$
s_{\text {relax }} \equiv s_{\text {creep }}=\frac{\mathrm{d} \sigma}{\mathrm{d} \ln \dot{\varepsilon}}=\frac{k T}{V}
$$

where $\dot{\varepsilon}$ is the strain rate and $V$ is the activation volume. With this model [6], activation energy can be calculated by using the elastic modulus of the crystal, beam constant and the dimensions of the specimen. This method is, however, complicated and requires measurements of many physical entities. Remember that $s_{\text {relax }}$ is the slope of stress relaxation curves. The activation energy for stress relaxation [3] [4] to be calculated is given by

$$
u_{o} \sim k\left(T_{o}+A T^{2}\right)\left[m+2.3\left(\frac{\mathrm{d} \sigma_{o}}{\mathrm{~d} s}\right)\right] ; \mathrm{d} s \equiv \frac{\left(\sigma_{o}-\sigma\right)}{\Delta t}
$$


where $m=\ln \left(\frac{\dot{\varepsilon}_{o}}{\dot{\varepsilon}}\right) \approx 25$ is taken as the classical value, $T_{o}$ and $A$ are constants. This relation is good enough for a limited low temperature range, i.e., $T \leq 70 \mathrm{~K}$ to account for quantum mechanical effects.

Using the simple or single barrier stochastic model of logarithmic creep of Buckle and Feltham [7], the quantum behaviour and stochasticity of crystal plasticity for Peierls barrier in cubic metals were studied by Jafri et al. [8]. Their assumption that the Peierls barrier width during creep, preferably the logarithmic creep, at relatively low temperatures, i.e., $1.7 \mathrm{~K} \leq T \leq 300 \mathrm{~K}$, would remain unchanged. This confirms to the fact that the Peierls barrier width is a quantum width or quantum length [9] [10]. The Peierls width and height, during creep, respectively [8] [9] are given as

$$
\begin{aligned}
& \text { Peierls barrier width during cre } \\
& =\omega=2 \pi v^{\prime} \mu b\left[1-\frac{\mu}{(1-v) k T}\right]
\end{aligned}
$$

Peierls barrier height

$$
=P_{h}=4 \pi^{2} v^{\prime} \mu b^{2}\left[\frac{2 \mu}{k T}-(1-v)\right] a .
$$

The quantized Peirels barrier width or quantum length [8] [9] is

$$
Q_{l}=2 \pi v^{\prime} \mu b
$$

where in Equations (6)-(8), $v^{\prime}$ is the frequency of phonon, $v$ is the Poissons ratio of crystal, $b$ is the Burgers vector, $\mu$ is the shear modulus of cubic metals and $a$ is the lattice of cubic crystals. We shall apply these equations to develop new meaningful expressions for crystal yielding, plasticity or work hardening with stress relaxations curves, quantization of dislocations over Peierls barrier and depinning of dislocations from Peierls barrier in the form of logarithm flux per unit time, i.e., logarithms fluence.

\section{Results and Discussions}

\subsection{Quantum Mechanical Yielding in Metals at Relatively Low Temperatures}

The anomalies in the temperature dependance of yield stress of metals at low temperature were studied [11]. Usually such anomalies are experimentally reported in the temperature range of $\frac{\Theta}{200} \leq T \leq \frac{\Theta}{10}$, where $\Theta$ is

Debye temperature (follows quantum theory of the specific heat of metals) in kelvin. Raza [12] ascribed these anomalies to diverse stress relaxations profiles in the plasticity of crystals. The stresses relaxation in the temperature range, $50 \mathrm{~K}<T \leq 300 \mathrm{~K}$ shows Gaussian profiles, i.e.,

$$
\frac{\mathrm{d} \sigma}{\mathrm{d} s}=\exp \left[-\left(\Delta t \times \sigma_{y}\right)^{2}\right]
$$

where $\sigma_{y}$ is the yield stress in the same temperature range. At temperatures $10 \mathrm{~K}<T \leq 50 \mathrm{~K}$, stress concentration and narrowing of slips bands are considered as quantum mechanical effects. The stresses are quantized with frozen vibrational energies,i.e., of phonon. In this temperature range, a log-normal stress relaxation behaviour is observed, i.e.,

$$
\frac{\mathrm{d} \sigma}{\mathrm{d} s}=\ln \left(t+t_{o}\right) .
$$

A sudden escalation in the yield stress below about $10 \mathrm{~K}$, following a linear profile of stress relaxation and indeed of yield stress with a negative slope, can be ascribed to quantum elasticity as a manifestation of stress causing effects, i.e., $\sigma_{y}=-c T ; T \leq 10 \mathrm{~K}$, where $c$ is the slope of the straight line.

Now, we modify such observations [12] by using Equation (3), i.e., for $k T \equiv k \Theta \approx \hbar\left(\frac{u_{o}}{m b^{2}}\right)^{1 / 2}$, [2] where $m$ is considered as the mass of phonon at different Debye temperature ranges. 


$$
\begin{gathered}
\sigma_{y}=\frac{1}{k \Theta} \simeq \frac{1}{\hbar}\left(\frac{m}{u_{o}}\right)^{1 / 2} b ; 50 \mathrm{~K}<\Theta \leq 300 \mathrm{~K} \\
\frac{\mathrm{d} \sigma}{\mathrm{d} s}=\ln \left(t+t_{o}\right), 10 \mathrm{~K}<\theta \leq 50 \mathrm{~K} \\
s=\frac{k T}{d V}=\frac{k \Theta}{d V}=\frac{\hbar}{b}\left(\frac{u_{o}}{m}\right)^{1 / 2} \cdot \frac{1}{V} .
\end{gathered}
$$

Using $s_{\text {relax }}=\frac{k \Theta}{V}$, we have

$$
\begin{gathered}
\sigma_{y} \equiv s=\frac{k \Theta}{V}=\frac{\hbar}{b}\left(\frac{u_{o}}{m}\right)^{1 / 2} \frac{1}{V} . \\
\Rightarrow \sigma_{y}=\frac{\hbar}{b}\left(\frac{u_{o}}{m}\right)^{1 / 2} \cdot \frac{1}{k \Theta} ; 10 \mathrm{~K}<\Theta \leq 50 \mathrm{~K} \\
\sigma_{y}=-c T \equiv-c \Theta=-c \frac{\hbar}{k b}\left(\frac{u_{o}}{m}\right)^{1 / 2} ; \Theta \leq 10 \mathrm{~K} .
\end{gathered}
$$

Equations (9)-(11) are self explanatory to reflect quantum mechanical yielding of crystals at different Debye temperatures.The quantum elasticity is confirmed from Equation (11) where pinned dislocations from Peierls barrier are stretched with a "quantum action, $\hbar$ " because the yield stress, $\sigma_{y}$ is $\hbar u_{o}^{1 / 2}$. The mass of phonon and the Bergers vector become smaller and more smaller with escalation of yield stress at temperature below 10 K. Equation (10) shows that the the yield stress becomes smaller and smaller with increasing, mass of the phonon and the activation volume. The quantization of stresses is evident from Equation (10). The yield stress in the Gaussian region, i.e., $50 \mathrm{~K}<\theta \leq 300 \mathrm{~K}$ is inversely proportional to “quantum action” $\hbar$, i.e., $\sigma_{y} \propto \frac{1}{\hbar u_{o}^{1 / 2}}$ and directly proportional to $\mathrm{bm}^{1 / 2}$.

\subsection{Quantum Mechanical Behaviour for Crystal Plasticity with Stress Relaxation Curves}

The logarithmic creep which Weertmann [2] obtained, is a tending profile towards quantum behaviour and so is the case of quantum mechanical tunneling of dislocations by Mott [1]. They could not ascertain the quantum mechanical behaviour of yield stress, plasticity with stress relaxation curves or logarithmic creep but their mathematical results are correct. We investigated quantum mechanical tunneling of dislocations by considering results of Mott [1], Weertmann [2], Jafri et al. [8], Majeed [9], Majeed et al. [10] and Raza [3]-[5] [11] [12]. Considering Equation (2) and Equation (4), together, i.e.,

$$
\begin{gathered}
\dot{\varepsilon}=\frac{N \hbar \sigma A}{10.4\left(M u_{o}\right)^{1 / 2} t} \text { and } \\
s_{\text {relax }} \equiv S_{\text {creep }}=\frac{\mathrm{d} \sigma}{\mathrm{d} \ln \epsilon}=\frac{k T}{V}=\frac{k \Theta}{V}
\end{gathered}
$$

Taking natural logarithm of Equation (2), we have

$$
\begin{aligned}
\dot{\varepsilon} & =\ln \frac{N \hbar \sigma A}{10.4\left(M u_{o}\right)^{1 / 2} t}=\ln N \hbar \sigma A-\ln \left[10.4\left(M u_{o}\right)^{1 / 2} t\right] \\
& \Rightarrow \ln \dot{\varepsilon}=\ln N \hbar \sigma A-\left[\ln 10.4+\ln \left(M u_{o}\right)^{1 / 2}+\ln t\right] \\
& =\ln N \hbar \sigma A-\ln 10.4-1 / 2 \ln (M u o)-\ln t=\ln \frac{N \hbar \sigma A}{t}-\ln 10.4-1 / 2 \ln (\text { Muo })
\end{aligned} .
$$


The last two terms in Equation (12) are explicit and have nothing to do with creep rate, therefore, they are neglected. Hence, we have

$$
\ln \dot{\varepsilon}=\ln \frac{N \hbar \sigma A}{t} .
$$

Putting Equation (13) in Equation (4), we have

$$
\begin{gathered}
S_{\text {relax }} \equiv S_{\text {creep }}=\frac{\mathrm{d} \sigma}{\mathrm{d} \ln \dot{\varepsilon}}=\frac{k \Theta}{V} \\
S_{\text {relax }}=\frac{\mathrm{d} \sigma}{\mathrm{d} \ln \frac{N \hbar \sigma A}{t}}=\frac{k \Theta}{V} .
\end{gathered}
$$

Equation (14) shows that the logarithmic creep rate is interpreted as stress relaxation rate ( stress relaxation curves in crystal plasticity). Equation (14) provides the slope, i.e., " $\mathrm{s}$ " of relaxation curve. Surprising all the relaxations curves when plotted and checked on semi-log or log-log graph papers shows the logarithmic profile which is a confirmation to the validity of the result of Mott [1] and Weertman [2]. Considering denominator of Equation (14), i.e., $\ln \frac{N \hbar \sigma A}{t}$ shows simultaneous quantization of dislocations over the Peierls barrier and depinning of dislocations from Peierls barrier in the form of fluence, i.e., flux/time. The term $\frac{N A}{t}$ in $\ln$ shows fluence, i.e., the number of depinned dislocations crossing the area per unit time whereas the term “ $\hbar \sigma$ ” in "ln" corresponds to quantization of dislocations with their stresses over the Peierls barrier, i.e.,

$$
s_{\text {relax }}=\frac{\mathrm{d} \sigma}{\mathrm{d}\left[\ln \hbar \sigma+\ln \frac{N A}{t}\right]}=\frac{k \Theta}{V} .
$$

\subsection{Interpretation of Quantum Length of Peierls Barrier}

With Equation (8) of quantized Peierls barrier width, i.e., $Q_{l}=2 \pi \eta v^{\prime} b$ where $v^{\prime}$ is the frequency, $\eta$ the shear modulus and $b$ the Burgers vector, we can ascertain that the frequency $v^{\prime}$ is associated with curvature of time, i.e., $\frac{\partial}{\partial t}$. The curvature of time which is an unitary operator will work on shear modulus, i.e., $\frac{\partial}{\partial t} \eta$ will provide the rate determining processes of dislocations (some of them quantized on Peierls barriers, whereas others are dipinned from Peierls barriers) in the curvature of space $\frac{\partial}{\partial r}$. This is how $\frac{\partial}{\partial t} \eta$ provides the activation energy values for both of the process at the quantum level. Remember that the curvatures of space $\frac{\partial}{\partial r}$ and time $\frac{\partial}{\partial t}$ are twinned with each other, i.e., $\nabla, \frac{\partial}{\partial t} \equiv \square$, which is a "dal” operator. For quantization of $Q_{l}=2 \pi \eta v^{\prime} b$ in the curvature of space, $\frac{\partial}{\partial r}$, the quantum action, h (Planck's constant)is to be replaced by $2 \pi \eta v^{\prime}$ equation

$$
Q_{l}=2 \pi \eta v^{\prime} b=h b
$$

where $h=2 \pi \eta v^{\prime} b$. Equation (16) shows the quantization of Burgers vector for crystals. When energy becomes oscillatory, the action is referred to as "quantum action". This is how space is configured which is known as "quanta", or wave packet. How Equation (8) is to be validated? Wherever and whenever, frequency at the quantum level is involved, there is a quantum action despite the fact that there is no $h$ (Planck's constant) or $\hbar$. There is a dire need to interpret $v^{\prime}$ in Equation (8). Bardoni Peakks [13] appear only on plastic deformation and due to dislocations. Bardoni peaks are observed in face and body centered cubic crystals. $v^{\prime}$, the frequency in Equation (8) can be replaced by Poisson's ratio because Bardoni like peaks (applied frequency equals the 
natural frequency, i.e., damped oscillations in crystal). Poission’s ratio, to our conjecture, is a ratio of applied strain rate to natural frequency of dislocations which are pinned about Peierls barrier under damped conditions (Poission, s ratio $\approx 1$ ). The over damping or under damping of dislocations from its pinning positions will cause the Poisson's ratio to change between zero and one, i.e., $0 \leq$ Poission's ratio, $v \leq 1$. With over damping the dislocation from Peierls barrier will be depinned and with under damping, the dislocations will be quantized with a curvature of space. This will cause the quantum length to appear in the form of torsion curvature, i.e.,

$$
Q_{l} \equiv \frac{\partial}{\partial(r, \theta)}=2 \pi \eta v
$$

where $v$ is Poission's ratio. With torsion curvature, Equation (17) will provide the line tension of shear modulus, $\eta$ which is also periodic in nature.

\section{Conclusion}

Theories of Mott and Weertmann pertaining to quantum mechanical tunneling of dislocations from Peierls barrier in cubic crystal are reconsidered. The quantum mechanical yielding in metals at relatively low temperature is resolved and formula is presented. The crystal plasticity is studied in terms of stress relaxation curves and the formula is presented. Formulas for simultaneous quantization of dislocations with their stress and depinning of dislocations are presented.

\section{References}

[1] Mott, N.F. (1956) Quantization of Dislocations Due to Lattice Vibrations in Cubic Crystals. Philosophical Magazine, 1, 568.

[2] Weertmann, J. (1958) Dislocations Crossing Peierls Barriers in Cubic Metals. Journal of Applied Physics Letters, 29, 1685.

[3] Raza, S.M. (1985) Stress Relaxation in Nickel At 15 to 300 K. Acta Metallurgica, 33, 1285-1291. http://dx.doi.org/10.1016/0001-6160(85)90239-1

[4] Raza, S.M. (1984) Yield Stress and Stress Relaxations Measurements in Cobalt. Journal of Applied Physics Letters, 55, 296-299. http://dx.doi.org/10.1063/1.333096

[5] Raza, S.M., Abidi, S.B.H. and Farooqui, N. (1988) Proposed Model for Stress Relaxation at Low Temperatures. Applied Physics Letters, 46, 34-35.

[6] Raza, S.M., Farooqui, N., Abidi, S.B.H., et al. (1987) Self-Consistent Stress Relaxation Model. Physica Status Solidi (a), 100, k149.

[7] Buckle, V.A. and Feltham, P. (1975) Single Barrier Stochastic Model of Logarithmic Creep in Cubic Crystals. Metal Science, 9, 541.

[8] Jafri, Y.Z., Farooqui, N., et al. (1996) Quantum Behaviour and Stochasticity, of Crystal Plasticity for Peierls Barrier in Cubic Crystals. Science International (Lahore), 8, 105-109.

[9] Majeed, S.A. (1994) Stochastic Models of Crystal Plasticity. M.Phil. Thesis, University of Balochistan, Quetta, Pakistan.

[10] Majeed, S.A., Farooqui, N. and Raza, S.M. (1995) Low Temperature Creep and Stress Relaxation for Peierls Barrier in Cubic Metals. Modern Physics Letters B, 9, 285-1995. http://dx.doi.org/10.1142/S0217984995000279

[11] Raza, S.M. (1983) Anomalies in the Yielding of Crystals at Relatively Low Temperatures. Canadian Journal of Physics, 61, 1527-1530.

[12] Raza, S.M. (2009) Quantum Mechanical Yielding in Metals at Relatively Low Temperatures. Science International (Lahore), 21, 151-153.

[13] Naborro, F.R.N. (1966) Theory of Crystal Dislocations. Oxford University Press, London, UK, 538. 